\title{
The role of $\beta$-arrestins in the termination and transduction of G-protein-coupled receptor signals
}

\author{
Louis M. Luttrell ${ }^{2,4}$ and Robert J. Lefkowitz ${ }^{1,2,3, *}$ \\ ${ }^{1}$ The Howard Hughes Medical Institute and the ${ }^{2}$ Departments of Medicine and ${ }^{3}$ Biochemistry, Duke University Medical Center, Durham, NC 27710, \\ USA \\ ${ }^{4}$ The Geriatrics Research, Education and Clinical Center, Durham Veterans Affairs Medical Center, Durham, NC 27705, USA \\ *Author for correspondence (e-mail: lefko001@receptor-biol.duke.edu) \\ Journal of Cell Science 115, 455-465 (2002) (C) The Company of Biologists Ltd
}

\begin{abstract}
Summary
$\beta$-arrestins are versatile adapter proteins that form complexes with most G-protein-coupled receptors (GPCRs) following agonist binding and phosphorylation of receptors by G-protein-coupled receptor kinases (GRKs). They play a central role in the interrelated processes of homologous desensitization and GPCR sequestration, which lead to the termination of $G$ protein activation. $\beta$ arrestin binding to GPCRs both uncouples receptors from heterotrimeric $G$ proteins and targets them to clathrincoated pits for endocytosis. Recent data suggest that $\beta$ arrestins also function as GPCR signal transducers. They can form complexes with several signaling proteins, including Src family tyrosine kinases and components of the ERK1/2 and JNK3 MAP kinase cascades. By recruiting
\end{abstract}

these kinases to agonist-occupied GPCRs, $\beta$-arrestins confer distinct signaling activities upon the receptor. $\beta$ arrestin-Src complexes have been proposed to modulate GPCR endocytosis, to trigger ERK1/2 activation and to mediate neutrophil degranulation. By acting as scaffolds for the ERK1/2 and JNK3 cascades, $\beta$-arrestins both facilitate GPCR-stimulated MAP kinase activation and target active MAP kinases to specific locations within the cell. Thus, their binding to GPCRs might initiate a second wave of signaling and represent a novel mechanism of GPCR signal transduction.

Key words: $\beta$-arrestin, G-protein-coupled receptor, Desensitization, Sequestration, Tyrosine kinase, Mitogen-activated protein kinase

\section{Introduction}

Initial attempts to study the role of G-protein-coupled receptor kinase (GRK) 2 in desensitization of $\beta 2$ adrenergic receptors in purified reconstituted systems produced a paradoxical finding: as the purity and specific activity of the kinase for agonist-occupied receptors is increased, its ability to inactivate receptor-Gs coupling declines. The finding that GRK2mediated $\beta 2$ adrenergic receptor desensitization can be restored by the addition of pure retinal arrestin, a $48 \mathrm{kDa}$ protein previously shown to mediate the rapid uncoupling of rhodopsin from the retinal heterotrimeric $\mathrm{G}$ protein, transducin, led to the hypothesis that additional arrestin-like proteins existed (Benovic et al., 1987). The loss of an essential cofactor during GRK2 purification, subsequently identified as $\beta$-arrestin 1 , accounted for the loss of GRK2-dependent desensitization by the highly purified kinase preparations.

To date, four functional members of the arrestin gene family have been cloned (Freedman and Lefkowitz, 1996; Ferguson, 2001). Two arrestins, visual arrestin (Shinohara et al., 1987; Yamaki et al., 1987) and cone arrestin (Murakami et al., 1993; Craft et al., 1994), are expressed almost exclusively in the retina, where they regulate photoreceptor function. The $\beta$ arrestins, $\beta$-arrestin 1 (Lohse et al., 1990) and $\beta$-arrestin 2 (Attramandal et al., 1992), are ubiquitously expressed proteins whose highest levels of expression are in the brain and spleen. All members of the family can bind specifically to lightactivated or agonist-occupied heptahelical G-protein-coupled receptors (GPCRs) that have been phosphorylated by GRKs. Arrestin binding sterically blocks the receptor-G-protein interaction and thus plays a critical role in the process of homologous desensitization, the specific uncoupling of agonist-bound GPCRs from their cognate G proteins.

$\beta$-arrestins, in addition to their role in GPCR desensitization, have additional functions not shared with the visual arrestins. By binding to components of the cellular endocytic machinery, $\beta$-arrestins act as adapter proteins that target GPCRs to clathrin-coated pits for endocytosis. The process of GPCR sequestration is important not only in attenuating GPCR signaling in the continued presence of agonist but also for receptor resensitization and downregulation. In addition, $\beta$-arrestins might have novel functions as GPCR signal transducers. Recent reports suggest that they bind directly to several proteins involved in signal transduction, including Src family kinases and components of the ERK1/2 and JNK3 MAP kinase cascades. By recruiting these proteins directly to the GPCR, $\beta$-arrestins can confer distinct enzymatic activities upon the receptor, which may lead to signals that are important for the regulation of cellular growth or differentiation. Here, we review recent advances in our understanding of the role of $\beta$-arrestins in GPCR signaling, both as terminators of G-protein-dependent signaling processes and as potential transducers of novel signals emanating from GPCRs.

\section{$\beta$-arrestins as signal terminators}

The waning of GPCR signaling in the continued presence of agonist is accomplished by a coordinated series of events that are typically considered as three distinct processes: receptor desensitization, sequestration and downregulation. 


\section{(1) Desensitization}

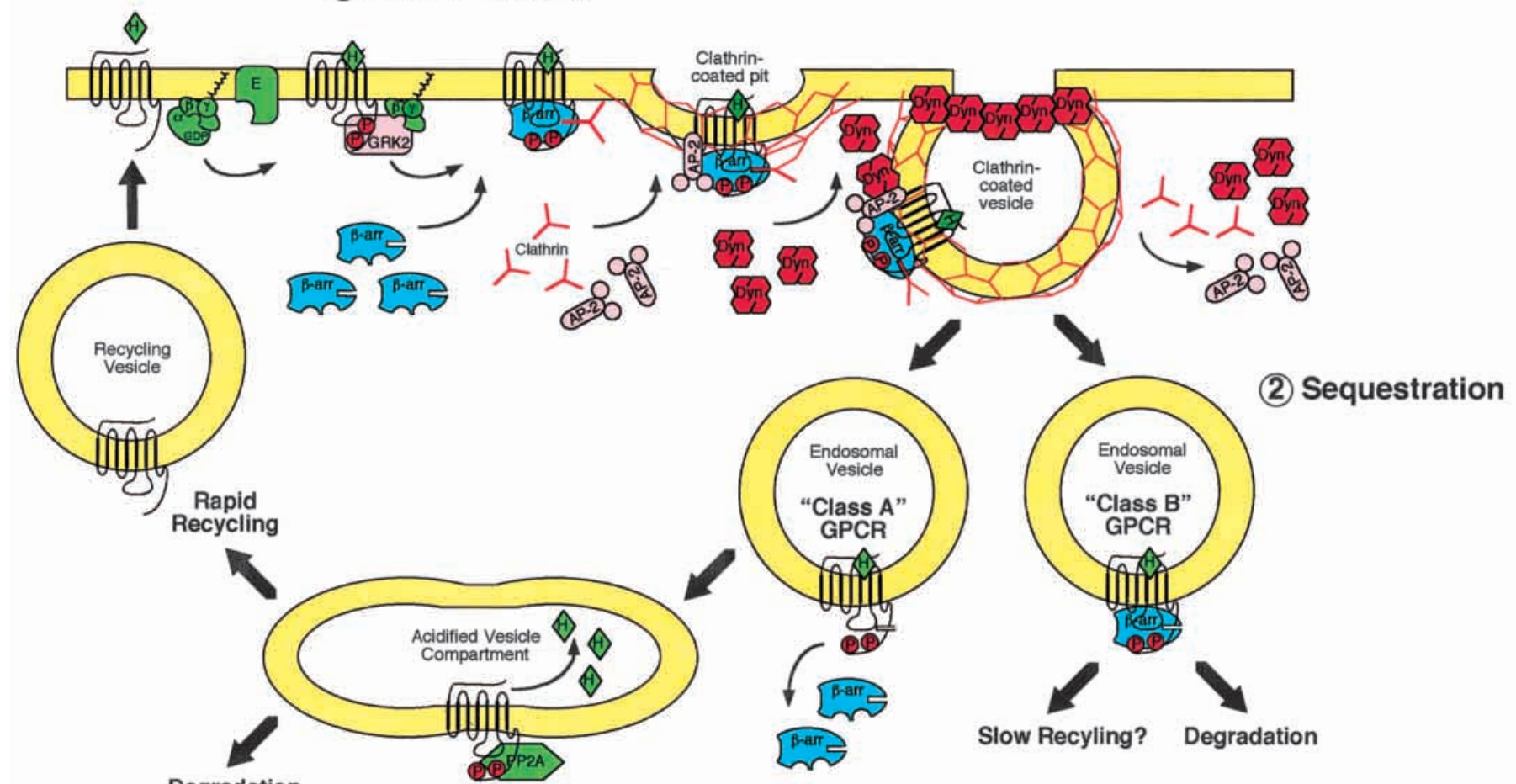

Degradation

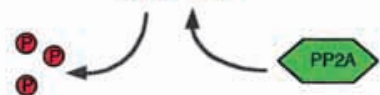

(3) Recycling / Downregulation
Fig. 1. Role of $\beta$-arrestins in the desensitization, sequestration and intracellular trafficking of GPCRs. Homologous desensitization of GPCRs (1) results from the binding of $\beta$-arrestins $(\beta$-arr) to agonist $(\mathrm{H})$-occupied receptors following phosphorylation of the receptor by GRKs. $\beta$-arrestin binding sterically precludes coupling between the receptor and

heterotrimeric $\mathrm{G}$ proteins, leading to termination of signaling by $\mathrm{G}$ proteins effectors (E). Receptor-bound $\beta$-arrestins also act as adapter proteins, binding to components of the clathrin endocytic machinery including clathrin, $\beta 2$-adaptin (AP-2) and NSF. Receptor sequestration (2) reflects the dynamin (Dyn)-dependent endocytosis of GPCRs via clathrin-coated pits. Once internalized, GPCRs exhibit two distinct patterns of $\beta$-arrestin interaction. 'Class A' GPCRs, for example the $\beta 2$ adrenergic receptor, rapidly dissociate from $\beta$-arrestin upon internalization. These receptors are trafficked to an acidified endosomal compartment, wherein the ligand is dissociated and the receptor dephosphorylated by a GPCR-specific protein phosphatase PP2A isoform, and are subsequently recycled to the plasma membrane (3). 'Class B' receptors, for example the angiotensin II AT1a receptor, form stable receptor- $\beta$-arrestin complexes. These receptors accumulate in endocytic vesicles and are either targeted for degradation or slowly recycled to the membrane via as yet poorly defined routes.

\section{$\beta$-arrestins in GPCR desensitization}

Desensitization, which begins within seconds of agonist exposure, is initiated by phosphorylation of the receptor. Second-messenger-dependent protein kinases, including cyclic-AMP-dependent protein kinase (PKA) and protein kinase $\mathrm{C}(\mathrm{PKC})$, phosphorylate serine and threonine residues within the cytoplasmic loops and $\mathrm{C}$-terminal tail domains of many GPCRs. Phosphorylation of these sites is sufficient to impair receptor-G-protein coupling efficiency in the absence of $\beta$-arrestins. For example, phosphorylation of the $\beta 2$ adrenergic receptor in vitro by PKA is sufficient to impair receptor-stimulated GTPase activity (Benovic et al., 1985), and removal of the PKA phosphorylation sites delays the onset of desensitization in intact cells (Bouvier et al., 1988). Agonist occupancy of the target GPCR is not required for this process, thus receptors that have not bound agonist, including receptors for other ligands, can be desensitized by the activation of second-messenger-dependent protein kinases. This lack of requirement for receptor occupancy has led to the use of the term heterologous desensitization to describe the process (Lefkowitz, 1993).

In contrast, homologous desensitization is mediated by phosphorylation of the receptor by GRKs and subsequent binding of $\beta$-arrestin (Fig. 1). There are seven known GRKs. Rhodopsin kinase (GRK1) and GRK7, a candidate for a cone opsin kinase (Weiss et al., 1998), are retinal kinases involved in the regulation of rhodopsin photoreceptors, whereas GRK2GRK6 are more widely expressed. Membrane targeting of all of the GRKs is apparently critical to their function and is conferred by a C-terminal tail domain (Stoffel et al., 1997). GRK1 and GRK7 each possess a C-terminal CAAX motif. Light-induced translocation of GRK1 from the cytosol to the plasma membrane is facilitated by the post-translational farnesylation of this site. The $\beta$-adrenergic receptor kinases (GRK2 and GRK3) have C-terminal G $\beta \gamma$-subunit-binding and pleckstrin-homology domains, and they translocate to the membrane as a result of interactions between these domains and free $G \beta \gamma$ subunits and inositol phospholipids. Palmitoylation of GRK4 and GRK6 on C-terminal cysteine residues leads to constitutive membrane localization. Targeting of GRK5 to the membrane is thought to involve the electrostatic interaction of a highly basic 46 residue $\mathrm{C}$-terminal domain with membrane phospholipids.

In common with second-messenger-dependent protein 
Fig. 2. Putative domain architecture of the $\beta$-arrestins. By analogy with the known crystal structure of visual arrestin, $\beta$-arrestins are thought to be composed of two major structural domains, $\mathrm{N}$ and $\mathrm{C}$, each comprising a seven-stranded $\beta$ sandwich. Based upon mutagenesis studies performed using both $\beta$-arrestins and visual arrestin, the $\beta$-arrestins are comprised of two major functional domains, an N-terminal (A) domain responsible for recognition of activated GPCRs and a C-terminal (B) domain responsible for secondary receptor recognition. The $\mathrm{A}$ and $\mathrm{B}$ domains are separated by a phosphate sensor domain $(\mathrm{P})$. The functionally identified A and B domains correspond approximately to the $\mathrm{N}$ and $\mathrm{C}$ domains identified crystallographically. N (R1)- and C (R2)-terminal regulatory domains reside at either end of the protein. The R2 domain contains the primary site of $\beta$-arrestin 1 phosphorylation, S412, as well as the LIEF binding motif for clathrin and the RXR binding motif for $\beta 2$-adaptin (AP2). The recognition domain for inositol phospholipids (IP6) resides within the B domain. One or more PXXP motifs located within the A domain of $\beta$-arrestin 1 mediates binding to the c-Src-SH3 domain. The MAP kinase, JNK3, and possibly other MAP kinases (MAPKs), interact with $\beta$-arrestin 2 via a consensus MAP kinase recognition sequence, RRSLHL, located within the B domain. Less precisely defined interactions, such as those between $\beta$-arrestin 1 (1-185) and Ask1 and Src-SH1 domains, $\beta$-arrestin 1 and NSF, and $\beta$-arrestin 2 and Mdm2, are also shown. Regions of the protein involved in receptor or membrane recognition are shown in blue, those involved in controlling $\beta$-arrestin interaction with the endocytic machinery are shown in red, while proposed interactions between $\beta$-arrestins and signaling proteins are shown in green.

kinases, GRKs phosphorylate GPCRs on serine and threonine residues in their third intracellular loop and C-terminal domains. GRKs, however, preferentially phosphorylate receptors that are in the agonist-occupied conformation. Furthermore, GRK phosphorylation alone has little effect on receptor-G-protein coupling in the absence of arrestins. Rather, the role of GRK phosphorylation is to increase the affinity of the receptor for arrestins. In vitro, the $\beta$-arrestin-1binding affinity of $\beta 2$ adrenergic receptors is increased 10- to 30-fold following phosphorylation of the receptor by GRK2 (Lohse et al., 1993). It is the binding of arrestin to receptor domains involved in $G$ protein coupling, rather than GRK phosphorylation, that leads to homologous desensitization of the receptor.

The crystal structure of visual arrestin indicates that arrestins contain two major domains, an $\mathrm{N}$ domain (residues 8-180) and a C domain (residues 188-362), each of which is composed of a seven stranded $\beta$ sandwich (Graznin et al., 1998; Hirsch et al., 1999) (Fig. 2). Mutagenesis studies performed on visual arrestin suggest that the $\mathrm{N}$ domain contains regions of the molecule that are important for recognition of light-activated rhodopsin, whereas a secondary-receptor-binding region resides within the $\mathrm{C}$ domain (Gurevich et al., 1995). Additional regulatory motifs reside at the $\mathrm{N}$ - and $\mathrm{C}$-termini of the molecule. A phosphate sensor region localizes to the linker between the $\mathrm{N}$ and $\mathrm{C}$ domains and forms part of the polar core of the protein. Interactions between the $\mathrm{C}$-terminal tail and the phosphate sensor region that maintain arrestin in an inactive state are disrupted upon receptor binding, allowing arrestin to bind with high affinity to the phosphorylated receptor.

The importance of $\beta$-arrestins in the regulation of GPCR function in vivo is indicated by data obtained from $\beta$-arrestinknockout mice. Homozygous $\beta$-arrestin-1-knockout animals are developmentally normal and exhibit normal resting cardiac parameters, such as heart rate, blood pressure and left ventricular ejection fraction. However, the administration of $\beta$-adrenergic receptor agonists produces an exaggerated hemodynamic response, which suggests that $\beta$-arrestin 1 plays a role in cardiac $\beta$-adrenergic receptor desensitization (Conner at al., 1997). Homozygous $\beta$-arrestin-2-knockout mice are likewise phenotypically normal, but exhibit a dramatic potentiation and prolongation of the analgesic effect of morphine, which is consistent with impaired $\mu$ opioid receptor desensitization in the central nervous system (Bohn et al., 1999). In these animals, the loss of opioid receptor desensitization correlates with an inability to develop tolerance to the antinociceptive effects of morphine, but does not prevent the development of opioid dependence (Bohn et al., 2000).

\section{$\beta$-arrestins in GPCR sequestration}

Internalization of GPCRs, also termed receptor sequestration or endocytosis, occurs more slowly than desensitization, happening over a period of several minutes after agonist exposure. It is now clear that GRK-mediated GPCR phosphorylation and binding of $\beta$-arrestin to the receptor facilitates the agonist-promoted endocytosis of many GPCRs, including the $\beta 2$ adrenergic, angiotensin II type 1a, m2-m5 muscarinic cholinergic, endothelin A, D2 dopamine, follitropin, monocyte chemoattractant protein-1, CCR-5 and CXCR1 receptors (Ferguson, 2001) (Fig. 1). The extent of $\beta$ arrestin involvement appears to vary significantly depending on the receptor, agonist and cell type, probably reflecting a variation in endogeous patterns of GRK and $\beta$-arrestin expression, the specific effects of agonist and partial agonist drugs on receptor conformation and the availability of alternative pathways for GPCR endocytosis.

The physical basis for the differential effects of visual arrestins and $\beta$-arrestins on GPCR endocytosis apparently resides within the $\mathrm{C}$-terminal tail of the molecule (Fig. 2). $\beta$ arrestins contain two motifs that allow them to function as adapter proteins that link the GPCR to components of the clathrin-dependent endocytic machinery. $\beta$-arrestins, but not visual arrestins, bind with high affinity and stoichiometry to clathrin in vitro (Goodman et al., 1996). This interaction involves binding of an LIEF sequence, which is located 
between residues 374 and 377 of $\beta$-arrestin 2 (Krupnick et al., 1997), to a region located between amino acids 89 and 100 of the N-terminal domain of the clathrin heavy chain. In addition, $\beta$-arrestins bind directly to the $\beta 2$ adaptin subunit of the heterotetrameric AP-2 adaptor complex through an RxR sequence, which is located between residues 394 and 396 of $\beta$-arrestin 2 (Laporte et al., 1999; Laporte et al., 2000). The AP-2 complex links many receptors to the clathrin endocytic machinery by binding to clathrin, dynamin and EPS-15, and is involved in the initiation of clathrin-coated pit formation (Kirchhausen, 1999). Mutation of the AP-2-binding motif of $\beta$-arrestin 2 does not prevent it from binding to agonistoccupied $\beta 2$ adrenergic receptors but does block the targeting of receptor- $\beta$-arrestin complexes to clathrin-coated pits (Laporte et al., 2000). Expression of a dominant inhibitory mutant of dynamin, a large GTPase necessary for the fission of clathrin-coated pits from the plasma membrane, impairs the endocytosis of $\beta 2$ adrenergic receptors (Zhang et al., 1996), which supports the hypothesis that clathrin-coated pits mediate the endocytosis of many GPCRs.

The N-ethylmaleimide-sensitive fusion protein (NSF) also binds to $\beta$-arrestin 1 in vitro and in vivo (McDonald et al., 1999). NSF is an ATPase involved in intracellular transport. Overexpression of NSF enhances $\beta 2$ adrenergic receptor endocytosis in HEK-293 cells, which suggests that the interaction between $\beta$-arrestin and NSF is important for receptor endocytosis. $\beta$-arrestin activity is further influenced by binding of phosphoinositides, in particular InsP6 (Gaidarov et al.,1999). The phosphoinositide-binding region of $\beta$-arrestin 2 resides within residues 233-251. Mutation of basic residues within this region produces a $\beta$-arrestin that translocates to the membrane but fails to target $\beta 2$ adrenergic receptors to clathrin-coated pits, thereby inhibiting endocytosis of the receptor.

The endocytic function of $\beta$-arrestin 1 is also apparently regulated by phosphorylation. Cytoplasmic $\beta$-arrestin 1 is almost stoichiometrically phosphorylated on S412 (Lin et al., 1997). Upon translocation to the membrane, $\beta$-arrestin 1 is rapidly dephosphorylated. An S412D mutant of $\beta$-arrestin 1 that mimics the phosphorylated state binds agonist-occupied $\beta 2$ adrenergic receptors and mediates desensitization, but binds poorly to clathrin, thereby inhibiting receptor sequestration. Dephosphorylation of S412, which lies within the C-terminal regulatory domain, must therefore be necessary for the receptor- $\beta$-arrestin complex to engage the endocytic machinery. Interestingly, the kinases responsible for phosphorylation of $\beta$-arrestin 1 appear to be ERK1 and ERK2 (Lin et al., 1999). Since $\beta$-arrestins can form macromolecular complexes with activated ERKs (DeFea et al., 2000a; DeFea et al., 2000b; Luttrell et al., 2001), leading to localization of the kinases to specific intracellular compartments, phosphorylation of S412 may represent a mechanism of feedback regulation of ERK function.

While $\beta$-arrestin 2 is not phosphorylated at its C-terminus, its endocytic function is regulated by post-translational modification after binding to the receptor. Both $\beta$-arrestin 2 and $\beta 2$ adrenergic receptors have recently been shown to undergo rapid, $\beta$-arrestin-dependent ubiquitination in response to agonist binding (Shenoy et al., 2001). Ubiquitination of $\beta$ arrestin 2 is catalyzed by the E3 ubiquitin ligase Mdm2, which binds directly to the $\beta$-arrestin. Ubiquitination of the receptor is carried out by an as yet unidentified ubiquitin ligase. $\beta$ arrestin ubiquitination is apparently required for receptor internalization, whereas ubiquitination of the receptor is involved in degradation of the receptor but not its internalization.

Work with purified proteins in vitro and in overexpression systems has revealed little in terms of functional differences between the two $\beta$-arrestins. However, recent studies of fibroblast lines derived from mouse embryos (MEFs) lacking either or both $\beta$-arrestins confirm the hypothesis that $\beta$-arrestin 1 and $\beta$-arrestin 2 exhibit functional specialization (Kohout et al., 2001). Desensitization of both $\beta 2$ adrenergic and angiotensin AT1a receptors is impaired in both $\beta$-arrestin 1 and $\beta$-arrestin 2 knockout MEFs and further reduced in the doubleknockout cells, which suggests that the two isoforms are equally effective at inducing desensitization. In contrast, $\beta 2$ adrenergic receptor sequestration is markedly reduced only in $\beta$-arrestin-2-knockout and double-knockout MEFs, not in $\beta$ arrestin 1 knockouts. Reconstitution of $\beta$-arrestin expression in double-knockout MEFs revealed that $\beta$-arrestin 2 is 100 -fold more potent than $\beta$-arrestin 1 in supporting $\beta 2$ adrenergic receptor endocytosis. AT1a receptor sequestration is minimally affected in $\beta$-arrestin 1 knockouts and markedly impaired only in the double-knockout MEFs. This suggests that either $\beta$ arrestin alone is sufficient for AT1a receptor sequestration.

\section{$\beta$-arrestins in GPCR downregulation and resensitization}

Downregulation of GPCRs, the persistent loss of cell surface receptors that occurs over a period of hours to days, is the least understood of the processes controlling GPCR responsiveness. Control of cell surface receptor density occurs at least partially at the transcriptional level, but the removal of agonist-occupied receptors from the cell surface and their sorting for either degradation or recycling to the membrane is also important, at least in the early stages of downregulation (Fig. 1). Consistent with this hypothesis is the finding that downregulation of $\beta 2$ adrenergic receptors does not occur in $\beta$-arrestin- $1 / \beta$-arrestin2 double-knockout MEFs (Kohout et al., 2001).

Resensitization of a GPCR requires its dephosphorylation and dissociation from its ligand. Several lines of evidence support the hypothesis that receptor internalization is required for resensitization of many GPCRs (Sibley et al., 1986; Ferguson, 2001). Preventing GPCR endocytosis either by pharmocological means, such as treatment with concanavalin A or hypertonic sucrose, or by using mutant receptors that can signal and become desensitized, but exhibit defective endocytosis has demonstrated that sequestration is required for resensitization. In COS-7 cells, overexpression of $\beta$-arrestins enhances the rate of $\beta 2$ adrenergic receptor resensitization, which indicates that $\beta$-arrestin-dependent endocytosis plays a role in the process (Zhang et al., 1997).

Shortly after stimulation, phosphorylated $\beta 2$ adrenergic receptors appear in an endosomal vesicle fraction that is enriched in GPCR-specific protein phosphatase PP2A activity (Pitcher et al., 1995). Dephosphorylation of the receptor occurs in an acidified vesicle compartment, as treatment of cells with ammonium chloride, which neutralizes the acidity of endosomal vesicles, blocks association of the receptor with the phosphatase and prevents receptor dephosphorylation (Krueger et al., 1997). 


\section{(1) Dynamin Phosphorylation}

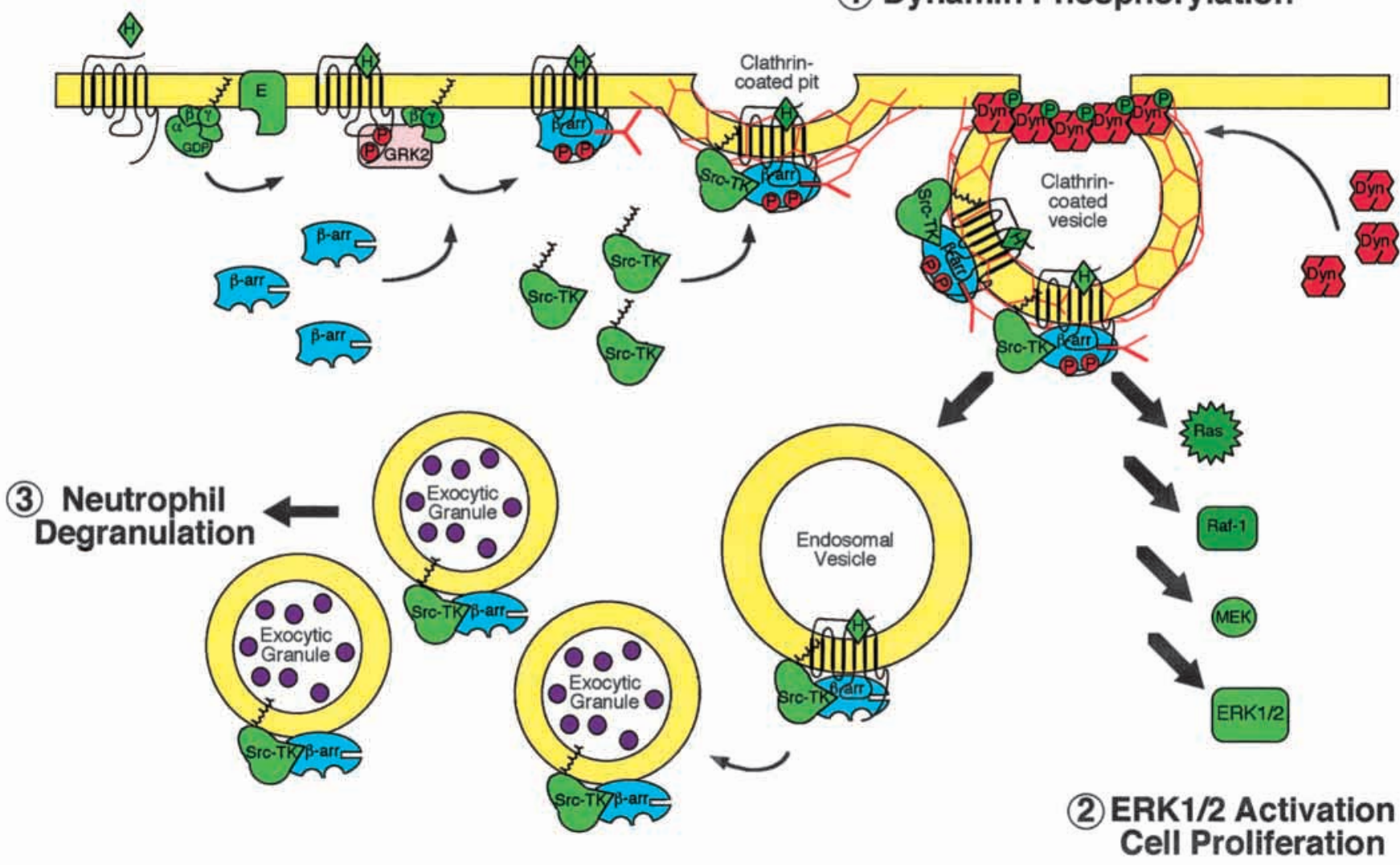

Fig. 3. Proposed roles of $\beta$-arrestin-dependent recruitment of Src kinases in GPCR signaling. The binding of $\beta$-arrestins to agonist-occupied GPCRs coincides with the recruitment of Src family tyrosine kinases, including c-Src, Hck and c-Fgr (Src-TK), to the receptor- $\beta$-arrestin complex. Several signaling events have been reported to involve $\beta$-arrestin-dependent Src recruitment. These include the regulation of clathrindependent $\beta 2$-adrenergic receptor endocytosis by tyrosine phosphorylation of dynamin (1), Ras-dependent activation of the ERK1/2 MAP kinase cascade and stimulation of cell proliferation by $\beta 2$-adrenergic and neurokinin NK1 receptors (2), and stimulation of chemokine CXCR1 receptor-mediated neutrophil degranulation (3).

Our understanding of the role of $\beta$-arrestins in determining the ultimate fate of internalized GPCRs has been advanced by the use of GFP-tagged $\beta$-arrestin chimeras that permit visualization of $\beta$-arrestin and receptor trafficking in live cells (Barak et al., 1997). Using this approach, Oakley et al. have recently shown that GPCRs exhibit different patterns of agonist-induced $\beta$-arrestin interaction, which allow the receptors to be grouped into two distinct classes (Oakley et al., 2000) (Fig. 1). Class A receptors include the $\beta 2$ and $\alpha 1 \mathrm{~B}$ adrenergic, $\mu$ opioid, endothelin $\mathrm{A}$ and dopamine D1A receptors. These receptors bind to $\beta$-arrestin 2 with higher affinity than $\beta$-arrestin 1 and do not bind to visual arrestin. In addition, their interaction with $\beta$-arrestin is transient. $\beta$-arrestin is recruited to the receptor at the plasma membrane and translocates with it to clathrin-coated pits; however, the receptor- $\beta$-arrestin complex dissociates upon internalization of the receptor, such that, as the receptor proceeds into an endosomal pool, the $\beta$-arrestin recycles to the plasma membrane (Zhang et al., 1999). Class B receptors, represented by the angiotensin AT1a, neurotensin 1, vasopressin 2, thyrotropin-releasing hormone and neurokinin NK-1 receptors, bind to $\beta$-arrestin 1 and $\beta$-arrestin 2 with equal affinity and also interact with visual arrestin. These receptors form stable complexes with $\beta$-arrestin, such that the receptor- $\beta$-arrestin complex internalizes as a unit that is targeted to endosomes.
The structural features of the receptor that dictate the stability of the receptor- $\beta$-arrestin complex reside within specific clusters of serine and threonine residues in the $\mathrm{C}$-terminal tail of the receptor (Oakley et al., 2001). The C-terminus of $\beta$ arrestin also determines the stability of the interaction, since a $\beta$-arrestin mutant truncated at residue 383 binds to the $\beta 2$ adrenergic receptor (a class A GPCR) with high affinity and traffics with it into endosomes.

The stability of the receptor- $\beta$-arrestin interaction might dictate the fate of the internalized receptor. The $\beta 2$ adrenergic receptor is rapidly dephosphorylated and recycled to the plasma membrane, whereas the vasopressin $\mathrm{V} 2$ receptor, a class $\mathrm{B}$ receptor, recycles slowly. Switching the C-terminal tails of these two receptors, which converts the $\beta 2$ adrenergic receptor into class $\mathrm{B}$ receptor and the $\mathrm{V} 2$ receptor into class $\mathrm{A}$ receptor, completely reverses the pattern of dephosphorylation and recycling (Oakley et al., 1999). Thus, the formation of a transient receptor- $\beta$-arrestin complex favors rapid dephosphoryation and return to the plasma membrane, whereas the formation of a stable receptor- $\beta$-arrestin complex retards resensitization and may favor targeting of the receptor for degradation. This role of $\beta$-arrestin may have important consequences. A naturally occurring loss-of-function mutation of the V2 receptor, $\mathrm{R} 137 \mathrm{H}$, which is associated with familial nephrogenic diabetes insipidus, is constitutively phosphorylated 
and localizes to $\beta$-arrestin-associated endosomal vesicles in the absence of agonist. Mutating the $\mathrm{R} 137 \mathrm{H}$ receptor to eliminate high-affinity $\beta$-arrestin binding re-establishes plasma membrane localization of the receptor and allows it respond to agonist (Barak et al., 2001).

\section{$\beta$-arrestins as signal transducers}

Recent data from yeast two-hybrid screens using $\beta$-arrestins and from biochemical characterization of receptor- $\beta$-arrestin complexes have contributed to the hypothesis that $\beta$-arrestins play roles in cellular signaling beyond their dampening effects on receptor-G-protein coupling. The finding that $\beta$-arrestins interact directly with Src family tyrosine kinases and components of the ERK1/2 and JNK3 MAP kinase modules suggests that $\beta$-arrestins may function more broadly as adapters to recruit signaling proteins to agonist-occupied GPCRs.

\section{$\beta$-arrestin-dependent recruitment of Src family kinases to GPCRs}

The initial evidence suggesting that $\beta$-arrestins function as tranducers of GPCR signals came from the observation that $\beta$ arrestins can bind directly to Src family kinases and recruit them to an agonist-occupied GPCR. In HEK-293 cells, stimulation of $\beta 2$ adrenergic receptors triggers the colocalization of the receptor with both endogenous $\beta$-arrestins and Src kinases in clathrin-coated pits (Luttrell et al., 1999). This colocalization reflects the assembly of a protein complex containing activated Src, $\beta$-arrestin and the receptor (Fig. 3). Similar results have been obtained in KNRK cells, in which $\beta$ arrestins are involved in recruiting Src to the neurokinin-1 receptor (DeFea et al., 2000b), and in neutrophils, in which $\beta$ arrestins recruit the Src family kinases Hck and Fgr to the CXCR-1 receptor (Barlic et al., 2000).

The binding of Src to $\beta$-arrestin 1 is mediated in part by an interaction between the Src homology (SH) 3 domain of the kinase and proline-rich PXXP motifs located at residues 88-91 and $121-124$ in the $\beta$-arrestin $1 \mathrm{~N}$ domain. A second major site of interaction involves the $\mathrm{N}$-terminal portion of the catalytic (SH1) domain of Src and additional epitopes located within the $\mathrm{N}$-terminal 185 residues of $\beta$-arrestin 1 (Miller et al., 2000). Binding to Src evidently does not preclude $\beta$-arrestin binding to the receptor, as all three proteins can be isolated as a complex following activation of $\beta 2$ adrenergic or neurokinin NK1 receptors (Luttrell et al., 1999; DeFea et al., 2000b).

$\beta$-arrestin-mediated Src recruitment has been implicated in several GPCR-mediated signaling events, including tyrosine phosphorylation of dynamin (Miller et al., 2000), activation of the ERK MAP kinase cascade (Luttrell et al., 1999; DeFea et al., 2000b) and stimulation of neutrophil degranulation (Barlic et al., 2000) (Fig. 3). Stimulation of $\beta 2$ adrenergic receptors results in the rapid Src-dependent tyrosine phosphorylation of dynamin (Ahn et al., 1999). Deletion of two Srcphosphorylation sites in dynamin I creates a dominant inhibitory form of the protein that impairs $\beta 2$ receptor endocytosis, which suggests that tyrosine phosphorylation represents a mechanism for modulating dynamin activity. Expression of a mutant Src protein that contains only a catalytically inactive SH1 domain but still binds avidly to $\beta$ - arrestin selectively inhibits $\beta 2$-adrenergic-receptor-stimulated tyrosine phosphorylation of dynamin and receptor internalization (Miller et al., 2000). These data suggest that one role of the $\beta$-arrestin-Src complex is to modulate GPCR endocytosis.

The Ras-dependent activation of the ERK1/2 MAP kinase pathway by many GPCRs requires Src kinase activity (Luttrell et al., 1996). In some cases, the interaction between $\beta$-arrestin and Src appears to be important for GPCR-mediated ERK1/2 activation. In HEK-293 cells, overexpression of $\beta$-arrestin 1 mutants that exhibit either impaired Src binding or are unable to target receptors to clathrin-coated pits blocks $\beta 2$ adrenergic receptor-mediated activation of ERK1/2 (Luttrell et al., 1999). In KNRK cells, activation of NK1 receptor by substance P leads to assembly of a scaffolding complex containing the internalized receptor, $\beta$-arrestin, Src and ERK1/2. Expression of either a dominant-negative $\beta$-arrestin 1 mutant or a truncated NK1 receptor that fails to bind to $\beta$-arrestin blocks complex formation and inhibits both substance-P-stimulated endocytosis of the receptor and activation of ERK1/2 (DeFea et al., 2000).

In granulocytes, activation of the chemokine receptor CXCR 1 by IL- 8 stimulates the rapid formation of complexes containing endogenous $\beta$-arrestin and Hck or Fgr (Barlic et al., 2000). The formation of $\beta$-arrestin-Hck complexes leads to Hck activation and trafficking of the complexes to granule-rich regions. Granulocytes expressing a dominant-negative $\beta$ arrestin mutant that exhibits impaired Src binding fail to activate tyrosine kinases. In these cells, chemoattractantstimulated granule release after IL-8 stimulation is inhibited, which suggests that $\beta$-arrestin-Hck complexes play a key role in the trafficking of exocytic vesicles.

\section{$\beta$-arrestins as MAP kinase scaffolds}

The MAP kinases are a family of evolutionarily conserved serine/threonine kinases that are involved in the transduction of externally derived signals regulating cell growth, division, differentiation and apoptosis. Mammalian cells contain at least three major classes of MAP kinase: ERKs, JNKs (also known as Stress-activated protein kinase, SAPK) and p38/HOG1 MAP kinases. The ERK pathway is important for control of the G0-G1 cell cycle transition and the passage of cells through mitosis or meiosis. In contrast, the JNK/SAPK and p38/HOG1 MAP kinases are involved in regulation of growth arrest, apoptosis and activation of immune and reticuloendothelial cells in response to a variety of environmental and hormonal stresses (Kryiakis and Avruch, 1996; Pearson et al., 2001).

MAP kinase activity in cells is regulated by a series of parallel kinase cascades comprising three kinases that successively phosphorylate and activate the downstream component. In the ERK1/2 cascade, for example, the proximal kinases, Raf-1 and B-Raf (MAP kinase kinase kinases), phosphorylate and activate MEK1 and MEK2 (MAP kinase kinases). MEK1 and MEK2 are dual function threonine/ tyrosine kinases that, in turn, carry out the phosphorylation and activation of ERK1/2. Although all of the intermediates are not fully characterized, the regulation of the JNK/SAPK and p38/HOG1 MAP kinase modules also involves a MAP kinase kinase kinase, MAP kinase kinase and MAP kinase phosphorylation cascade. Once activated, MAP kinases 


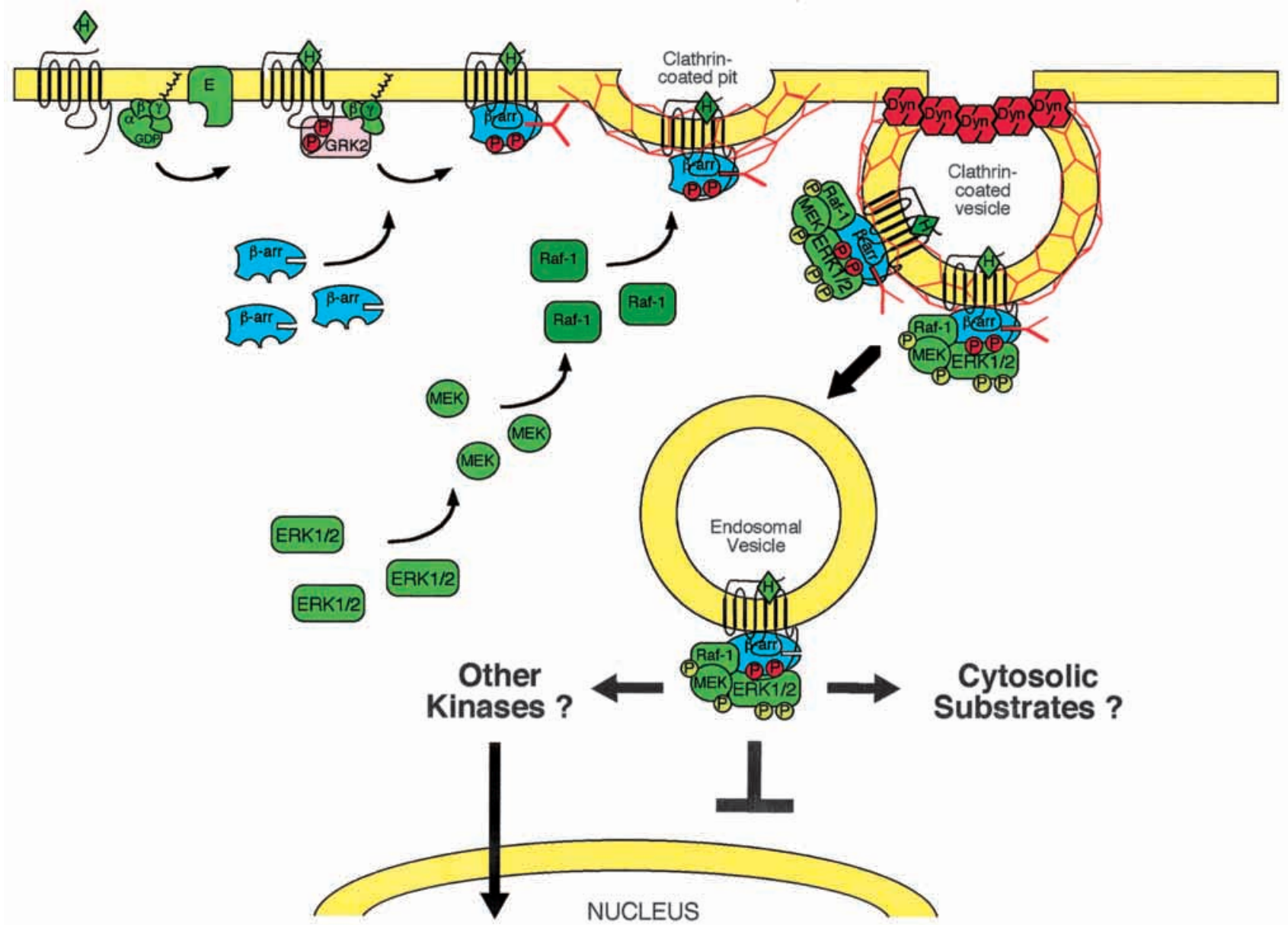

Fig. 4. Proposed role of $\beta$-arrestins in the activation and targeting of MAP kinases. The binding of $\beta$-arrestins to agonist-occupied GPCRs triggers the assembly of a MAP kinase activation complex using $\beta$-arrestin as a scaffold, with subsequent activation of a $\beta$-arrestin-bound pool of ERK1/2. The receptor- $\beta$-arrestin-ERK complexes are localized to endosomal vesicles, and their formation does not result in nuclear translocation of activated ERK1/2 or stimulation of cell proliferation. The function of $\beta$-arrestin-bound ERK1/2 is presently unknown. Activation of ERK1/2 by $\beta$-arrestin scaffolds may favor the phosphorylation of plasma membrane, cytosolic, or cytoskeletal ERK1/2 substrates, or it may lead to transcriptional activation through the ERK-dependent activation of other kinases. The model depicts $\beta$-arrestin scaffolding of the ERK1/2 MAP kinase cascade, based upon data obtained with the protease-activated PAR2 and angiotensin AT1a receptors. A similar mechanism has been proposed for regulation of the JNK3 MAP kinase cascade by AT1a receptors.

phosphorylate a variety of membrane, cytoplasmic, nuclear and cytoskeletal substrates. Upon activation, these kinases translocate to the nucleus, where they phosphorylate and activate nuclear transcription factors involved in DNA synthesis and cell division (Pearson et al., 2001).

In many cases, activation of MAP kinase cascades is controlled by binding of the component kinases to a scaffolding protein (Burack and Shaw, 2000; Pearson et al., 2001). These scaffolds serve at least three functions in cells: to increase the efficiency of signaling between successive kinases in the phosphorylation cascade; to ensure signaling fidelity by dampening cross talk between parallel MAP kinase cascades; and to target MAP kinases to specific subcellular locations. The prototypic MAP kinase scaffold is the Saccharomyces cervisiae protein Ste $5 p$ (Elion, 2001). In the yeast pheromone mating pathway, Ste 5p binds to Ste11p (a MAP kinase kinase kinase), Ste7p (a MAP kinase kinase) and to either Fus3p or Kss1p (MAP kinases) (Choi et al., 1994). Binding of yeast mating factor to the pheromone receptor, a GPCR, triggers heterotrimeric $G$ protein activation. Subsequent translocation of Ste $5 \mathrm{p}$ to the plasma membrane in response to the release of G $\beta \gamma$ subunits leads to activation of the Fus3/Kss1 cascade. Although no structural homologues of Ste $5 p$ have thus far been isolated from mammalian cells, several mammalian proteins that can bind to two or more components of a MAP kinase module, and might perform analogous scaffolding functions, have been identified. For example, the JIP family of proteins act as scaffolds for regulation of the JNK/SAPK pathway (Whitmarsh et al., 1998; Yasuda et al., 1999).

Recent data suggest that $\beta$-arrestins can function as scaffolds for some MAP kinase modules (Fig. 4). Stimulation of proteinase-activated receptor 2 (PAR2) receptor in KNRK cells induces the assembly of multiprotein complexes containing the internalized receptor, $\beta$-arrestin 1, Raf-1 and activated ERK1/2 (DeFea et al., 2000b). Complex assembly is apparently required for activation of ERK by the wild-type PAR2 receptor, since it is blocked by expression of a truncated form of $\beta$-arrestin that inhibits receptor endocytosis. The complexes also appear to function in the targeting of ERK, as the $\beta$-arrestin-ERK complexes are retained in the cytosol. Qualitatively similar 
results have been obtained for the angiotensin II type 1a (AT1a) receptor expressed in HEK293 and COS-7 cells (Luttrell et al., 2001). AT1a receptor activation results in the formation of complexes containing AT1aR, $\beta$-arrestin 2 and the component kinases of the ERK cascade: cRaf-1, MEK1 and ERK2. Upon receptor internalization, activated ERK2 appears in the same endosomal vesicles that also contain AT1aR- $\beta$-arrestin complexes. The NK1 neurokinin receptor provides a third example. Activation of NK1 receptors by substance $\mathrm{P}$ causes the formation of complexes comprising internalized receptor, $\beta$ arrestin, Src and ERK1/2 (DeFea et al., 2000a). These data suggest that $\beta$-arrestins function as scaffolds for the ERK1/2 MAP kinase cascade. If so, they are unique among the mammalian MAP kinase scaffolds described thus far, in that, in common with Ste5p, their function is directly under the control of a cell surface receptor.

$\beta$-arrestins might also be involved in scaffolding other MAP kinase pathways. In whole brain lysates and yeast two-hybrid assays, $\beta$-arrestin 2 binds to the neuronal JNK/SAPK isoform, JNK3 (McDonald et al., 2000). When coexpressed in COS-7 cells, $\beta$-arrestin 2 forms complexes with the MAP kinase kinase kinase Ask1, the MAP kinse kinase MKK4 and JNK3 but not JNK1 or JNK2. Ask1 binds to the $\beta$-arrestin $2 \mathrm{~N}$ terminus, whereas JNK3 binding is conferred by an RRSLHL motif in the C-terminal half of $\beta$-arrestin 2 (MacDonald et al., 2001; Miller et al., 2001). This motif, which is not present in $\beta$-arrestin 1 , corresponds to a consensus MAP kinase binding motif that has been identified in several other MAP-kinasebinding proteins. Overexpression of $\beta$-arrestin 2 dramatically increases Ask1-dependent phosphorylation of JNK3. Moreover, $\beta$-arrestin 2 expression causes cytosolic retention of JNK3 and, following stimulation of AT1a receptors, both $\beta$ arrestin 2 and JNK3 colocalize to intracellular vesicles. Thus, $\beta$-arrestin 2 can also behave as a scaffold for the JNK3 MAP kinase cascade, bringing the activity and spatial distribution of this MAPK module under the control of a GPCR.

The ability of $\beta$-arrestins to control both the activity and spatial distribution of MAP kinases might have important functional implications. Many GPCRs simultaneously employ multiple distinct mechanisms to activate MAP kinases (Gutkind, 1998; Pierce et al., 2001). The angiotensin AT1aR receptor, for example, can activate ERK1/2 not only via $\beta$ arrestin-dependent pathways but also through G-proteindependent signals and crosstalk with classical receptor tyrosine kinases (Eguchi et al., 1998; Heeneman, 2000; Gschwind, 2001). The crosstalk between GPCRs and EGF receptors accounts for the proliferative response to GPCR stimulation in a number of systems (Murasawa et al., 1998; Castagliuolo et al., 2000). In contrast, $\beta$-arrestin-dependent ERK activation does not lead to proliferative signaling. Wild-type PAR2 receptors, which mediate $\beta$-arrestin-dependent activation of a predominantly cytosolic pool of ERK1/2 in KNRK cells, do not stimulate $3 \mathrm{H}$-thymidine incorporation or cell replication (DeFea et al., 2000b).

$\beta$-arrestin-ERK complexes appear to be relatively stable entities in that they can be isolated by both gel filtration and by immunoprecipitation (DeFea et al., 2000a; DeFea et al., 2000 b; Luttrell et al., 2001). Since $\beta$-arrestins are cytosolic proteins, the formation of stable complexes between $\beta$-arrestin and activated ERK probably leads to cytosolic retention of ERK1/2. Significantly, a mutant PAR2 receptor lacking GRK phosphorylation sites, which cannot bind to $\beta$-arrestin, can still activate ERK1/2 but does so through a mechanistically distinct $\mathrm{Ca}^{2+}$ - and Ras-dependent pathway. This receptor mutant, unlike the wild-type receptor, induces nuclear translocation of ERK and stimulates cell proliferation (DeFea et al., 2000b). Thus, the nature of the GPCR- $\beta$-arrestin interaction appears to dictate both the predominant mechanism of ERK activation and, thereby, the consequences of ERK activation.

Little is currently known about the functional role of $\beta$-arrestin-ERK complexes. In addition to directly phosphorylating nuclear transcription factors, ERK1/2 phosphorylates numerous plasma membrane, cytoplasmic and cytoskeletal substrates (Pearson et al., 2001). These include several proteins involved in heptahelical receptor signaling, such as $\beta$-arrestin 1 (Lin et al., 1999), GRK2 (Pitcher et al., 1999; Elorza et al., 2000) and GAIP (Ogier-Denis et al., 2000). One potential role of $\beta$-arrestin-ERK complex formation could be to specifically target ERK1/2 to non-nuclear substrates involved in the regulation of GPCR signaling or intracellular trafficking. Alternatively, $\beta$-arrestin-bound ERK1/2 might phosphorylate other cytosolic proteins involved in transcriptional regulation, such as p90RSK, which in turn relay signals to the nucleus. In such a model, transcriptional events mediated directly by the nuclear pool of ERK1/2 would be attenuated, whereas alternative pathways of ERK-dependent transcription would persist, resulting in an altered pattern of transcription following activation of the GPCR.

\section{Arrestins in growth, development and disease}

Mice lacking the genes for either $\beta$-arrestin 1 or $\beta$-arrestin 2 , despite demonstrated perturbations of GPCR desensitization, exhibit normal embryological development (Conner et al., 1997; Bohn et al., 1999). Whether this indicates that $\beta$-arrestins are not required for normal development or that the presence of one $\beta$-arrestin isoform is sufficient to compensate for the lack of the other is unclear. However, a homozygous knockout of both murine $\beta$-arrestins results in early embryonic lethality, a finding that suggests a requirement for arrestins in development. Better evidence comes from the knockout of the Drosophila kurtz gene, which encodes a novel nonvisual arrestin (Roman et al., 2000). Kurtz is expressed ubiquitously during early embryonic development and later localizes primarily to the central nervous system and fat bodies. Mutations in kurtz that severely reduce its function produce a broad lethal phase extending from late embryogenesis to the third larval instar that is characterized by the formation of melanotic tumors within the fat bodies. Expression of the kurtz gene within the CNS rescues the lethality. While it remains unclear whether the kurtz mutant phenotype reflects a requirement for arrestins for the termination of G-proteinmediated signals or for the transduction of arrestin-dependent signals, the data clearly support a critical role for arrestins in developmental regulation.

Alterations in visual arrestin function are associated with retinal disease in flies, mice and humans. Certain forms of hereditary stationary night blindness, such as Oguchi disease, are attributable to mutations in rhodopsin kinase or visual arrestin, that result in impaired photoreceptor desensitization (Nakazawa et al., 1998; Yamada et al., 1999; Dryja, 2000). Many of these patients progress to the development of retinitis 
pigmentosa with the death of photoreceptor cells. Arrestin knockout mice maintained in continuous or cyclic light, but not in continuous darkness, experience photoreceptor loss at a rate proportional to the amount of light exposure, consistent with the hypothesis that constitutive signal flow in the absence of arrestin leads to photoreceptor degeneration (Chen et al., 1999). A different mechanism, possibly involving arrestindependent signaling, has been demonstrated in several different retinal degeneration mutants in Drosophila (Alloway et al., 2000). In these models, the formation of stable arrestin-rhodopsin complexes leads to apoptotic death of photoreceptor cells, and deletion of either rhodopsin or arrestin rescues the degeneration phenotype. The retinal degeneration requires the endocytic machinery, suggesting that the endocytosis of rhodopsin-arrestin complexes might be a molecular mechanism for triggering the apoptotic pathway. In light of the data indicating that $\beta$-arrestin 2 can serve as a scaffold for the JNK3 pathway (McDonald et al., 2000), these results suggest that arrestin scaffolds may be involved in this form of retinal degeneration.

\section{Conclusions and perspectives}

The $\beta$-arrestins play a well established role in the termination of receptor-G-protein coupling. GRK phosphorylation and $\beta$ arrestin binding are central to the processes of homologous desenstization, sequestration, recycling and downregulation of most mammalian GPCRs. Moreover, $\beta$-arrestins are increasingly appreciated as potential GPCR signal transducers. Recent data suggest that, by acting as adapter proteins and scaffolds, $\beta$-arrestins confer novel signaling properties upon GPCRs. $\beta$-arrestin-dependent recruitment of Src family tyrosine kinases might modulate GPCR endocytosis, trigger ERK1/2 activation and regulate exocytosis. By acting as scaffolds for the ERK1/2 and JNK3 cascades, $\beta$-arrestins may facilitate GPCR-stimulated MAP kinase activation and target MAP kinases to specific locations within the cell. At present, little is known about the physiological significance of these novel $\beta$-arrestin-dependent signaling mechanisms. But given our growing appreciation of the role of GPCRs in the control of cell differentiation, growth and oncogenesis in physiological and pathophysiological states (Dhanasekaran et al., 1995), it appears likely that these novel signaling mechanisms will be found to have important functional consequences.

R.J.L. is an investigator with the Howard Hughes Medical Institute. This work was supported by National Institutes of Health Grants DK55524 (L.M.L.) and HL16037 (R.J.L.). We thank D. Addison, M. Holben and J. Turnbough for excellent secretarial assistance.

\section{References}

Ahn, S., Maudsley, S., Luttrell, L. M., Lefkowitz, R. J. and Daaka, Y. (1999). Src-mediated tyrosine phosphorylation of dynamin is required for beta2-adrenergic receptor internalization and mitogen-activated protein kinase signaling. J. Biol. Chem. 274, 1185-1188.

Alloway, P. G., Howard, L. and Dolph, P. J. (2000). The formation of stable rhodopsin-arrestin complexes induces apoptsis and photoreceptor cell degeneration. Neuron 28, 129-138.

Attramandal, H., Arriza, J. L., Aoki, C., Dawson, T. M., Codina, J., Kwatra, M. M., Snyder, S. H., Caron, M. G. and Lefkowitz, R. J. (1992). $\beta$-arrestin 2, a novel member of the arrestin/ $\beta$-arrestin gene family. J. Biol. Chem. 267, 17882-17890.
Barak, L. S., Ferguson, S. S., Zhang, J. and Caron, M. G. (1997). A betaarrestin/green fluorescent protein biosensor for detecting $\mathrm{G}$ protein-coupled receptor activation. J. Biol. Chem. 272, 27497-27500.

Barak, L. S., Oakley, R. H., Laporte, S. A. and Caron, M. G. (2001). Constitutive arrestin-mediated desensitization of a human vasopressin receptor mutant associated with nephrogenic diabetes insipidus. Proc. Natl. Acad. Sci. USA 98, 93-98.

Barlic, J., Andrews, J. D., Kelvin, A. A., Bosinger, S. E., DeVries, M. E., Xu, L., Dobransky, T., Feldman, R. D., Ferguson, S. S. G. and Kelvin, D. J. (2000). Regulation of tyrosine kinase activation and granule release through $\beta$-arrestin by CXCRI. Nat. Immunol. 1, 227-233.

Benovic, J. L., Pike, L. J., Cerione, R. A., Staniszewski, C., Yoshimasa, T., Codina, J., Caron, M. G. and Lefkowitz, R. J. (1985). Phosphorylation of the mammalian beta-adrenergic receptor by cyclic AMP-dependent protein kinase. Regulation of the rate of receptor phosphorylation and dephosphorylation by agonist occupancy and effects on coupling of the receptor to the stimulatory guanine nucleotide regulatory protein. J. Biol. Chem. 260, 7094-7101.

Benovic, J. L., Kuhn, H., Weyand, I., Codina, J., Caron, M. G. and Lefkowitz, R. J. (1987). Functional desensitization of the isolated betaadrenergic receptor by the beta-adrenergic receptor kinase. Potential role of an analog of the retinal protein arrestin (48-kDa protein). Proc. Natl. Acad. Sci. USA 84, 8879-8882.

Bohn, L. M., Lefkowitz, R. J., Gainetdinov, R. R., Peppel, K., Caron, M. G. and Lin, F.-T. (1999). Enhanced morphine analgesia in mice lacking beta-arrestin 2. Science. 286, 2495-2498.

Bohn, L. M., Gainetdinov, R. R., Lin, F.-T., Lefkowitz, R. J and Caron, M. G. (2000). Mu-opioid receptor desensitization by beta-arrestin-2 determines morphine tolerance but not dependence. Nature 408, 720-723.

Bouvier, M., Hausdorff, W. P., De Blasi, A., O'Dowd, B. F., Kobilka, B. K., Caron, M. G. and Lefkowitz, R. J. (1988). Removal of phosphorylation sites from the beta 2-adrenergic receptor delays onset of agonist-promoted desensitization. Nature. 333, 370-373.

Burack, W. R. and Shaw, A. S. (2000). Signal transduction: Hanging on a scaffold. Curr. Opin. Cell Biol. 12, 211-216.

Castagliuolo, I., Valenick, L., Liu, J. and Pothoulakis, C. (2000), Epidermal growth factor receptor transactivation mediates substance Pinduced mitogenic responses in U-373 MG cells. J. Biol. Chem. 275, 26545-26550.

Chen, J., Simon, M. I., Matthes, M. T., Yasumura, D. and LaVail, M. M. (1999). Increased susceptibility to light damage in an arrestin knockout mouse model of Oguchi disease (stationary night blindness). Invest. Ophthalmol. Vis. Sci. 40, 2978-2982.

Choi, K. Y., Satterberg, B., Lyons, D. M. and Elion, E. A. (1994). Ste5 tethers multiple protein kinases in the MAP kinase cascade required for mating in S. cerevisiae. Cell. 78, 499-512.

Conner, D. A., Mathier, M. A., Mortensen, R. M., Christe, M., Vatner, S. F., Seidman, C. E. and Seidman, J. G. (1997). Beta-arrestin 1 knockout mice appear normal but demonstrate altered cardiac responses to betaadrenergic stimulation. Circ. Res. 81, 1021-1026.

Craft, C. M., Whitmore, D. H. and Weichmann, A. F. (1994). Cone arrestin identified by targeting expression of a functional family. J. Biol. Chem. 269, 4613-4619.

DeFea, K. A., Vaughn, Z. D., O’Bryan, E. M., Nishijima, D., Dery, O. and Bunnett, N. W. (2000a). The proliferative and antiapoptotic effects of substance $P$ are facilitated by formation of a $\beta$-arrestin-dependent scaffolding complex. Proc. Nat. Acad. Sci. USA 97, 11086-11091.

DeFea, K. A., Zalevsky, J., Thoma, M. S., Dery, O., Mullins, R. D. and Bunnett, N. W. (2000b). $\beta$-arrestin-dependent endocytosis of proteinaseactivated receptor 2 is required for intracellular targeting of activated ERK1/2. J. Cell Biol. 148, 1267-1281.

Dhanasekaran, N., Heasley, L. E. and Johnson, G. L. (1995). G proteincoupled receptor systems involved in cell growth and oncognesis. Endocr. Rev. 16, 259-270.

Dryja, T. P. (2000). Molecular genetics of Oguchi disease, fundus albipunctatus, and other forms of stationary night blindness. Am. J. Ophthal. 130, 547-563.

Eguchi, S., Numaguchi, K., Iwasaki, H., Matsumoto, T., Yamakawa, T., Utsunomiya, H., Motley, E. D., Owada, K. M., Hirata, Y., Marumo, F. and Inagami, T. (1998). Calcium-dependent epidermal growth factor receptor transactivation mediates the angiotensin II-induced mitogenactivated protein kinase activation in vascular smooth muscle cells. J. Biol. Chem. 273, 8890-8896.

Elion, E. A. (2001). The STE5p scaffold. J. Cell Sci. 114, 3967-3978. 
Elorza, A., Sarnago, S. and Mayor, F., Jr (2000). Agonist-dependent modulation of $\mathrm{G}$ protein-coupled receptor kinase 2 by mitogen-activated protein kinases. Mol. Pharm. 57, 778-783.

Ferguson, S. S. (2001). Evolving concepts in G protein-coupled receptor endocytosis: The role in receptor desensitization and signaling. Pharm. Rev. 53, 1-24.

Freedman, N. J. and Lefkowitz, R. J. (1996). Desensitization of G proteincoupled receptors. Recent Prog. Horm. Res. 51, 319-351.

Gaidarov, I., Krupnick, J. G., Falck, J. R., Benovic, J. L. and Keen, J. H. (1999). Arrestin function in $\mathrm{G}$ protein-coupled receptor endocytosis requires phosphoinositide binding. EMBO J. 18, 871-881.

Goodman. O. B., Jr, Krupnick, J. G., Santini, F., Gurevich, V. V., Penn, R. B., Gagnon, A. W., Keen, J. H. and Benovic, J. L. (1996). Beta-arrestin acts as a clathrin adaptor in endocytosis of the beta2-adrenergic receptor Nature 383, 447-450.

Graznin, J., Wilden, U., Choe, H. W., Labahn, J., Krafft, B. and Buldt, G. (1998). X-ray crystal structure of arrestin from bovine rod outer segments. Nature 391, 918-921.

Gschwind, A., Zwick, E., Prenzel. N., Leserer, M. and Ullrich, A. (2001). Cell communication networks: epidermal growth factor receptor transactivation as the paradigm for interreceptor signal transmission. Oncogene 20, 1594-1600.

Gurevich, V. V., Dion, S. B., Onorato, J. J., Ptasienski, J., Kim, C. M., Sterne-Marr, R., Hosey, M. M. and Benovic, J. L. (1995). Arrestin interactions with $\mathrm{G}$ protein-coupled receptors. Direct binding studies of wild-type and mutant arrestins with rhodopsin, $\beta 2$-adrenergic, and $\mathrm{m} 2$ muscarinic cholinergic receptors. J. Biol. Chem. 270, 720-731.

Gutkind, J. S. (1998). The pathways connecting G protein-coupled receptors to the nucleus through divergent mitogen-activated protein kinase cascades. J. Biol. Chem. 273, 1839-1842.

Heeneman, S., Haendeler, J., Saito, Y., Ishida, M. and Berk B. C. (2000) Angiotensin II induces transactivation of two different populations of the platelet-derived growth factor beta receptor. Key role for the p66 adaptor protein Shc. J. Biol. Chem. 275, 15926-15932.

Hirsch, J. A., Schubert, C., Gurevich, V. V. and Sigler, P. B. (1999). The 2.8 A crystal structure of visual arrestin: A model for arrestin's regulation. Cell 97, 257-269.

Kirchhausen, T. (1999). Adapters for clathrin-mediated traffic. Annu. Rev. Cell. Dev. Biol. 15, 705-732.

Kohout, T. A., Lin, F.-T., Perry, S. J., Conner, D. A. and Lefkowitz, R. J. (2001). Beta-arrestin 1 and 2 differentially regulate heptahelical receptor signaling and trafficking. Proc. Natl. Acad. Sci. USA 98, 1601-1606.

Krueger, K. M., Daaka, Y., Pitcher, J. A. and Lefkowitz, R. J. (1997). The role of sequestration in $\mathrm{G}$ protein-coupled receptor resensitization. Regulation of beta2-adrenergic receptor dephosphorylation by vesicular acidification. J. Biol. Chem. 272, 5-8.

Krupnick, J. G., Goodman, O. B., Jr, Keen, J. H. and Benovic, J. L. (1997) Arrestin/clathrin interaction. Localization of the clathrin binding domain of nonvisual arrestins to the carboxy terminus. J. Biol. Chem. 272, 1501115016.

Kryiakis, J. M. and Avruch, J. (1996). Sounding the alarm: protein kinase cascades activated by stress and inflammation. J. Biol. Chem. 271, 2431324316.

Laporte, S. A., Oakley, R. H., Zhang, J., Holt, J. A., Ferguson, S. S., Caron, M. G. and Barak, L. S. (1999). The beta2-adrenergic receptor/beta-arrestin complex recruits the clathrin adaptor AP-2 during endocytosis. Proc. Natl. Acad. Sci. USA 96, 3712-3717.

Laporte, S. A., Oakley, R. H., Holt, J. A., Barak, L. S. and Caron, M. G. (2000). The interaction of beta-arrestin with the AP-2 adaptor is required for the clustering of beta 2 -adrenergic receptor into clathrin-coated pits. $J$. Biol. Chem. 275, 23120-23126.

Lefkowitz, R. J. (1993). G protein-coupled receptor kinases. Cell. 74, 409 412.

Lin, F.-T., Krueger, K. M., Kendall, H. E., Daaka, Y., Fredericks, Z. L., Pitcher, J. A. and Lefkowitz, R. J. (1997). Clathrin-mediated endocytosis of the beta-adrenergic receptor is regulated by phosphorylation/dephosphorylation of beta-arrestin 1. J. Biol. Chem. 272, 31051-31057

Lin, F.-T., Miller, W. E., Luttrell, L. M. and Lefkowitz, R. J. (1999). Feedback regulation of beta-arrestin 1 function by extracellular signalregulated kinases. J. Biol. Chem. 274, 15971-15974.

Lohse, M. J., Benovic, J. L., Codina, J., Caron, M. G. and Lefkowitz, R. J. (1990). $\beta$-arrestin: a protein that regulates $\beta$-adrenergic receptor function. Science. 248, 1547-1550.
Lohse, M. J., Andexinger, S., Pitcher, J., Trukawinski, S., Codina, J., Faure, J.-P., Caron, M. G. and Lefkowitz, R. J. (1993). Receptor specific desensitization with purified proteins. Kinase dependence and receptor specificity of $\beta$-arrestin and arrestin in the $\beta 2$-adrenergic receptor and rhodopsin systems. J. Biol. Chem. 267, 8558-8564.

Luttrell, L. M., Hawes, B. E., van Biesen, T., Luttrell, D. K., Lansing, T. J. and Lefkowitz, R. J. (1996). Role of c-Src in G protein-coupled receptorand G $\beta \gamma$ subunit-mediated activation of mitogen activated protein kinases J. Biol. Chem. 271, 19443-19450.

Luttrell, L. M., Ferguson, S. S. G., Daaka, Y., Miller, W. E., Maudsley, S., Della Rocca, G. J., Lin, F.-T., Kawakatsu, H., Owada, K., Luttrell, D. K. et al. (1999). $\beta$-Arrestin-dependent formation of $\beta 2$ adrenergic receptor/Src protein kinase complexes. Science. 283, 655-661.

Luttrell, L. M., Roudabush, F. L., Choy, E. W., Miller, W. E., Field, M. E., Pierce, K. L., Lefkowitz, R. J. (2001). Activation and targeting of extracellular signal-regulated kinases by $\beta$-arrestin scaffolds. Proc. Nat. Acad. Sci., USA 98, 2449-2454.

McDonald, P. H., Cote, N. L., Lin, F.-T., Premont, R. T., Pitcher, J. A. and Lefkowitz, R. J. (1999). Identification of NSF as a beta-arrestin 1-binding protein. Implications for beta2-adrenergic receptor regulation. J. Biol. Chem. 274, 10677-10680.

McDonald, P. H., Chow, C.-W., Miller, W. E., LaPorte, S. A., Field, M. E., Lin, F.-T., Davis, R. J. and Lefkowitz, R. J. (2000). B-Arrestin 2: a receptor-regulated MAPK scaffold for the activation of JNK3. Science $\mathbf{2 9 0}$, 1574-1577.

Miller, W. E., Maudsley, S., Ahn, S., Kahn, K. D., Luttrell, L. M. and Lefkowitz, R. J. (2000). $\beta$-Arrestin 1 interacts with the catalytic domain of the tyrosine kinase c-SRC. J. Biol. Chem. 275, 11312-11319.

Miller, W. E., McDonald, P. H., Cai, S. F., Field, M. F., Davis, R. J. and Lefkowitz, R. J. (2001). Identification of a motif in the carboxy terminus of $\beta$-arrestin 2 responsible for activation of JNK3. J. Biol. Chem. 276, $27770-27777$.

Murakami, A., Yajima, T., Sakuma, H., McClaren, M. J. and Inana, G. (1993). X-arrestin: a new retinal arrestin mapping to the X chromosome. FEBS Lett. 334, 203-209.

Murasawa, S., Mori, Y., Nozawa, Y., Gotoh, N., Shibuya, M., Masaki, H., Maruyama, K., Tsutsumi, Y., Moriguchi, Y., Shibazaki, Y. et al. (1998). Angiotensin II type 1 receptor-induced extracellular signal-regulated protein kinase activation is mediated by $\mathrm{Ca}^{2+} /$ calmodulin-dependent transactivation of epidermal growth factor receptor. Circ. Res. 82, 1338-1348.

Nakazawa, M., Wada, Y. and Tamai, M. (1998). Arrestin gene mutations in autosomal recessive retinitis pigmentosa. Arch. Ophthalmol. 116, 498501

Oakley, R. H., Laporte, S. A., Holt, J. A., Barak, L. S. and Caron, M. G. (1999). Association of beta-arrestin with G protein-coupled receptors during clathrin-mediated endocytosis dictates the profile of receptor resensitization. J. Biol. Chem. 274, 32248-32257.

Oakley, R. H., Laporte, S. A., Holt, J. A., Caron, M. G. and Barak, L. S. (2000). Differential affinities of visual arrestin, beta-arrestin 1, and betaarrestin 2 for $\mathrm{G}$ protein-coupled receptors delineate two major classes of receptors. J. Biol. Chem. 275, 17201-17210.

Oakley, R. H., Laporte, S. A., Holt, J. A., Barak, L. S. and Caron, M. G. (2001). Molecular determinants underlying the formation of stable intracellular $G$ protein-coupled receptor-beta-arrestin complexes after receptor endocytosis. J. Biol. Chem. 276, 19452-19460.

Ogier-Denis, E., Pattingre, S., EI Benna, J. and Codogno, P. (2000). Erk1/2dependent phosphorylation of Galpha-interacting protein stimulates its GTPase accelerating activity and autophagy in human colon cancer cells. $J$. Biol. Chem. 275, 39090-39095.

Pearson, G., Robinson, F., Beers Gibson, T., Xu, B.-E., Karandikar, M., Berman, K. and Cobb, M. H. (2001). Mitogen-activated protein (MAP) kinase pathways: regulation and physiologic functions. Endocr. Rev. 22, 153-183.

Pierce, K. L., Luttrell, L. M. and Lefkowitz, R. J. (2001). New mechanisms in heptahelical receptor signaling to mitogen activated protein kinase cascades. Oncogene 20, 1532-1539.

Pitcher, J. A., Payne, E. S., Csortos, C., DePaoli-Roach, A. A. and Lefkowitz, R. J. (1995). The G-protein-coupled receptor phosphatase: A protein phosphatase type $2 \mathrm{~A}$ with a distinct subcellular distribution and substrate specificity. Proc. Natl. Acad. Sci. USA 92, 8343-8347.

Pitcher, J. A., Tesmer, J. J., Freeman, J. L., Capel, W. D., Stone, W. C. and Lefkowitz, R. J. (1999). Feedback inhibition of G protein-coupled receptor kinase 2 (GRK2) activity by extracellular signal-regulated kinases. J. Biol. Chem. 274, 34531-34534. 
Roman, G., He, J. and Davis, R. L. (2000). Kurtz, a novel nonvisual arrestin, is an essential neural gene in Drosophila. Genetics 155, 1281-1295.

Shenoy, S. K., McDonald, P. H., Kohout, T. A. and Lefkowitz, R. J. (2001). Regulation of receptor fate by ubiquitination of activated $\beta 2$-adrenergic receptor and $\beta$-arrestin. Science 294, 1307-1313.

Shinohara, T., Dietzschold, B., Craft, C. M., Wistow, G., Early, J. J., Donoso, L. A., Horwitz, J. and Tao, R. (1987). Primary and secondary structure of bovine retinal S antigen (48-kDa protein). Proc. Natl. Acad. Sci. USA 84, 6975-6979.

Sibley, D. R., Strasser, R. H., Benovic, J. L., Daniel, K. and Lefkowitz, R. J. (1986). Phosphorylation/dephosphorylation of the beta-adrenergic receptor regulates its functional coupling to adenylate cyclase and subcellular distribution. Proc. Natl. Acad. Sci. USA 83, 9408-9412.

Stoffel, R. H., Pitcher, J. A. and Lefkowitz, R. J. (1997). Targeting G proteincoupled receptor kinases to their membrane substrates. J. Membr. Biol. 157, $1-8$.

Weiss, E. R., Raman, D., Shirakawa, S., Ducceschi, M. H., Bertram, P. T., Wong, F., Kraft, T. W. and Osawa, S. (1998). The cloning of GRK7, a candidate cone opsin kinase, from cone- and rod-dominant mammalian retinas. Mol. Vis. 4, 27.

Whitmarsh, A. J., Cavanagh, J., Tournier, C., Yasuda, J. and Davis, R. J.
(1998). A mammalian scaffold complex that selectively mediates MAP kinase activation. Science 281, 1671-1674.

Yamada, T., Matsumoto, M., Kadoi, C., Nagaki, Y., Hayasaka, Y. and Hayasaka, S. (1999). $1147 \mathrm{del}$ A mutation in the arrestin gene in Japanese patients with Oguchi disease. Ophthal. Genetics 20, 117-120.

Yamaki, K., Takahashi, Y., Sakuragi, S. and Matsubara, K. (1987). Molecular cloning of the S-antigen cDNA from bovine retina. Biochem. Biophys. Res. Commun. 142, 904-910.

Yasuda, J., Whitmarsh, A. J., Cavanagh, J., Sharma, M. and Davis, R. J. (1999). The JIP group of mitogen-activated protein kinase scaffold proteins. Mol. Cell. Biol. 19, 7245-7254.

Zhang, J., Ferguson, S. S., Barak, L. S., Menard, L. and Caron, M. G. (1996). Dynamin and beta-arrestin reveal distinct mechanisms for $\mathrm{G}$ proteincoupled receptor internalization. J. Biol. Chem. 271, 18302-18305.

Zhang, J., Barak, L. S., Winkler, K. E., Caron, M. G. and Ferguson, S. S. (1997). A central role for $\beta$-arrestins and clathrin-coated vesicle-mediated endocytosis in $\beta 2$-adrenergic receptor resensitization. J. Biol. Chem. 272, 27005-27014.

Zhang, J., Barak, L. S., Anborgh, P. H., Laporte, S. A., Caron, M. G. and Ferguson, S. S. (1999). Cellular trafficking of G protein-coupled receptor/beta-arrestin endocytic complexes. J. Biol. Chem. 274, 10999-11006. 